\title{
Dosimetric comparison of different algorithms in stereotactic body radiation therapy (SBRT) plan for non-small cell lung cancer (NSCLC)
}

This article was published in the following Dove Press journal: OncoTargets and Therapy

\author{
Jun Zhang ${ }^{l, *}$ \\ Dazhen Jiang ${ }^{1} * *$ \\ Huanfan $\mathrm{Su}^{2}$ \\ Zhitao $\mathrm{Dai}^{3}$ \\ Jing Dai ${ }^{1}$ \\ Hui Liu' \\ Conghua Xie' \\ Haijun Yu' \\ 'Department of Radiation and Medical \\ Oncology, Zhongnan Hospital of Wuhan \\ University, Hubei Cancer Clinical Study \\ Center, Hubei 430072, People's Republic \\ of China; ${ }^{2}$ Jiangxi Medical College, \\ Department of Medical Imaging, \\ Shangrao, Jiangxi 334000, People's \\ Republic of China; ${ }^{3}$ School of Physics and \\ Technology, Wuhan University, Wuhan \\ 43007I, People's Republic of China \\ *These authors contributed equally to \\ this work
}

\begin{abstract}
Purposes: The main aim of the study was to investigate the dosimetric difference between acuros $\mathrm{XB}$ algorithm (AXB), anisotropic analytic algorithm (AAA), and pencil beam convolution (PBC) algorithm in stereotactic body radiation therapy (SBRT) plan for non-small cell lung cancer (NSCLC).

Patients and Methods: Thirty-eight NSCLC patients were included. GTV, PTV, and organs at risk were delineated by the radiation oncologists. Three optimized SBRT plans for each patients were gained using three algorithms of AXB, AAA, and $\mathrm{PBC}$ with the identical plan parameters. Dosimetric endpoints were collected and compared among the three plans, including dosimetric criteria: V100\%, V90\%, PTV $\mathrm{D}_{\min }, \mathrm{D}_{\max }, \mathrm{D}_{\text {mean }}$, homogeneity index (HI), and Paddick conformity index (CI).

Results: AXB plan resulted in decreased $\mathrm{V} 100 \%$ with a mean difference $6.14 \%$ compared with $\mathrm{PBC}$ plan (For $\mathrm{V} 100 \%$, AXB vs AAA vs $\mathrm{PBC}=93.44 \%$ vs $95.54 \%$ vs $99.58 \%, P<0.05$ ). Three plans showed no significant difference as to the parameter V90\%. AXB plan leaded to reduced $\mathrm{D}_{\min }$ of PTV compared with other two algorithms (For $\mathrm{D}_{\min }$ of PTV, AXB vs AAA vs $\mathrm{PBC}=4048 \mathrm{cGy}$ vs $4365 \mathrm{~Gy}$ vs $4873 \mathrm{~Gy}, P<0.05$ ). $\mathrm{PBC}$ induced the enhanced trend of $\mathrm{D}_{\max }$ of PTV compared with other two algorithms ( $\mathrm{D}_{\max }$ among three algorithms, $P>0.05$ ); and increased the $\mathrm{D}_{\text {mean }}$ of PTV in three algorithms with significant difference (For $\mathrm{D}_{\text {mean }}$ of PTV, AXB vs AAA vs $\mathrm{PBC}=5332 \mathrm{cGy}$ vs $5330 \mathrm{~Gy}$ vs $5785 \mathrm{~Gy}, P<0.05$ ). AXB algorithm achieved a similar plan conformity with other two algorithms (For CI, AXB vs AAA vs $\mathrm{PBC}=0.80$ vs 0.85 vs $0.71, P>0.05$ ).
\end{abstract}

Conclusion: For SBRT plan of NSCLC, AAA and PBC algorithms overestimate target coverage, AXB algorithm is recommended for the SBRT plan of NSCLC.

Keywords: NSCLC, SBRT, AXB, AAA, PBC, dosimetric difference

\section{Introduction}

Stereotactic body radiation therapy (SBRT) has become a very important treatment technique for early stage non-small cell lung cancer, ${ }^{1,2}$ which delivers high radiation doses to a tumor target in a hypo-fractionated schedule with the minimum dose to the tissue around the target area. Therefore, it can protect the normal tissues and organs around the target. However, for non-homogeneous tissues such as lung, the usage of SBRT technology was challenged with the problem of accurate dose calculation. ${ }^{3}$ Therefore, SBRT of non-small cell lung cancer needs a more precise algorithm, which takes into account tissue non-uniformity correction to ensure the accuracy of dose calculation. Varian Eclipse Treatment Planning System (Varian Medical Systems, 
Palo Alto, USA) is a widely used treatment planning system in the current radiotherapy physics program with different versions. The latest Eclipse Treatment Planning System (Version 13.5) is equipped with Acuros XB (AXB) algorithm. However, a more precise $\mathrm{AXB}$ algorithm is not widely used due to many factors. ${ }^{4-10}$ Most of the studies only focus on the differences of algorithms in motifs. In order to intuitively understand the difference between different algorithms in the practice of patient planning, here a systematic comparison between $\mathrm{AXB}$, anisotropic analytic algorithm (AAA), and pencil beam convolution (PBC) on dosimetric differences of SBRT in non-small cell lung cancer was studied with the aim to provide references for the treatment planning of clinical radiotherapy.

\section{Patients and methods}

\section{Patients selection and target volume delineation}

The study was approved by the Ethics Committee of Zhongnan Hospital of Wuhan University. All patients signed informed consent prior to SBRT treatment, which included allowing their data to be used for further research. Owing to the retrospective nature of the present study, it was granted an exemption in writing by the Ethics Committee of Zhongnan Hospital of Wuhan. A total of 38 patients with non-small cell lung cancer who received SBRT from February 2014 to June 2018 in Zhongnan Hospital of Wuhan University were included. Thirtyeight patients consisted of 27 males and 11 females, with a median age of 65 years (range, 30-81 years). Thirty-five patients were stage IVB, and three patients were stage IA (According to the AJCC 2017 staging). These patients with stage IV had already received systemic chemotherapy or targeted therapy before receiving the treatment of SBRT. All of them experienced a progress of the disease or cannot continue to afford the other systemic therapy such as the third- generation TKI or immunotherapy due to the bad social economy status. The location of tumor target was as follows: 16 cases in the right upper lung, 4 cases in the right lower lung, 13 cases in the left upper lung, and 5 cases in left lower lung. The mean GTV volume is $24.08 \mathrm{~cm}^{3}$ (range, $0.5-114.1 \mathrm{~cm}^{3}$ ). Patient simulation was positioned using the whole-body immobilization system (R624-SCF, Klarity, Guangzhou, China). CT simulation images with $3 \mathrm{~mm}$ thickness were gained with a large aperture simulation CT scanner (Siemens Somatom Sensation, Munich, German) for each patients. Tumor motion was assessed with four-dimensional CT imaging with Varian real-time position management (Varian Medical Systems, Palo Alto, CA). The abdominal compression was employed for all patients. CT images were transferred to Eclipse version 13.5 planning system for target volume delineation and plan design. Gross tumor volume and internal target volume were segmented on a four-dimensional CT scan, respectively. A 5-mm uniform margin from ITV was typically used for creation of the PTV. 5 Organs at risk (OARs) were contoured including the heart, the spinal cord, the chest wall, the lung and so on. The same attending radiation oncologists delineated the target volumes and OARs. SBRT plans were generated by the same senior medical physicist.

\section{SBRT planning}

Varian Eclipse TPS (Version 13.5) was introduced to calculate all plans. Linear accelerator Clinac IX (Varian Medical Systems, Palo Alto, USA) was used for the radiotherapy treatment. The prescription dose of 38 patients for all tumors was 50 Gy in 5 fraction at the $90 \%$ isodose line of the maximum dose delivered every other day. All the dose limits to the critical organs are according to the protocol guidelines of the two trials (RTOG0813 and RTOG0618) and the recommendations of the American Association of Physicists in Medicine Task Group 101 report. The recommended maximal dose to the trachea and proximal bronchial tree was 30, 35, and 40 Gy for 3-, 4-, and 5-fraction regimens, respectively. The recommended esophageal maximal dose ranged from 25.2 to 35 Gy, and maximal heart dose ranged from 30 to $38 \mathrm{~Gy}$, increasing with the number of fractions used. For 5-fraction regimens, mediastinal structures were permitted to have a maximal point dose of $105 \%$ of the prescription dose. All plans were originally calculated with AAA algorithm, the computational grid was $1.5 \mathrm{~mm} \dot{\times} 1.5 \mathrm{~mm}$, and all plans are corrected for non-uniformity, then with the $\mathrm{AXB}$ and $\mathrm{PBC}$ algorithms with the same prescription dose and field parameters. Each plan met with the requirements of the RTOG 0813 Report. ${ }^{5}$ One hundred percent of the prescription dose includes at least $95 \%$ of PTV, $90 \%$ of the prescription dose included at least $99 \%$ of PTV.

\section{Evaluation of dosimetry}

In this study, the target coverage (V100\% and V90\% of the PTV), Conformal index (CI), Homogeneity index (HI), minimum, maximum, and average dose of PTV $\left(\mathrm{D}_{\min }, \mathrm{D}_{\max }\right.$, $\mathrm{D}_{\text {mean }}$ ) were compared. V100\% was defined as the volume 
of the prescription dose line for $100 \%$ includes the volume of PTV, V90\% was defined as the volume of PTV contained in the prescription dose line for $90 \%$, and the volume was normalized to the volume of the target area PTV. CI was calculated with Paddick Conformal index: $\mathrm{CI}_{\text {paddick }}=\frac{\left(\mathrm{TV} \mathrm{PI}_{\mathrm{PI}}\right)^{2}}{\mathrm{PI} * \mathrm{TV}}$, PI was the volume included in the prescription dose line, TV was the volume of PTV, TV $\mathrm{TI}_{\mathrm{PI}}$ was the volume of the prescription dose line including the volume of the PTV. A perfect plan was $\mathrm{TV}=\mathrm{PI}=\mathrm{TV}_{\mathrm{PI}}$ and $\mathrm{CI}_{\text {Paddick }}=1$. HI was calculated according to the ICRU 83 Report: $\mathrm{HI}=\frac{\mathrm{D}_{2 \%}-\mathrm{D}_{98 \%}}{\mathrm{D}_{50 \%}}$, $\mathrm{D}_{2 \%}$ was the maximum dose of volume accepted by $2 \%$ of PTV, D $_{98 \%}$ was the maximum dose of volume accepted by $98 \%$ of PTV, $\mathrm{D}_{50 \%}$ was the maximum dose of volume accepted by $50 \%$ of PTV. The value of the HI indicates the uniform dose distribution. When $\mathrm{HI}=0$ indicates that there is no drop in the target area, the uniformity is the best.

\section{Statistical analysis}

Data were analyzed using the Statistical Package for Social Sciences version 22.0 (IBM Corporation, Armonk, NY, USA). The significance of differences between mean values was determined by one-way ANOVA (Bonferroni test). $P$-values $<0.05$ were considered statistically significant.

\section{Results}

\section{Clinical characteristics}

Patient and tumor characteristics of the 38 patients are described in Table 1.

\section{VI $00 \%$ and $\mathrm{V} 90 \%$ of the PTV}

For 38 patients, the average values and standard viations are summarized in Table 2 and Figure 1 for V100\% and V $90 \%$ of the PTV. AXB plan resulted in decreased V100\% with a mean difference $6.14 \%$ compared with $\mathrm{PBC}$ plan (For $\mathrm{V} 100 \%$, AXB vs AAA vs $\mathrm{PBC}=93.44 \%$ vs 95.54 vs $99.58 \%, P<0.05)$. Three plans showed no significant

Table I Patient and tumor characteristics

\begin{tabular}{|l|l|}
\hline Parameter & Total \\
\hline Patients $(\mathrm{n}=38)$ & Female =I I, male =27 \\
Median age in years (range) & $65(30-8 \mathrm{I})$ \\
Median GTV in cm ${ }^{3}$ (range) & $24.08(0.5-||$ I I I) \\
Tumor location ( $\mathrm{n}=38)$ & I3 LUL, I6 RUL, 5 \\
& LLL, 4 RLL \\
\hline
\end{tabular}

Abbreviations: LUL, left upper lobe; RUL, right upper lobe; LLL, left lower lobe; RLL, right lower Lobe. difference as to the parameter V90\% (For V90\%, AXB vs $\mathrm{AAA}$ vs $\mathrm{PBC}=98.66 \%$ vs $99.75 \%$ vs $99.83 \%, P>0.05)$.

\section{$D_{\text {min }}, D_{\text {max }}$, and $D_{\text {mean }}$ of PTV}

$\mathrm{D}_{\text {min }}, \mathrm{D}_{\text {max }}$, and $\mathrm{D}_{\text {mean }}$ of PTV in 38 patients are indicated in Table 3 and Figure 2. AXB plan leaded to reduced $D_{\text {min }}$ of PTV compared with other two algorithms (For $\mathrm{D}_{\min }$ of PTV, AXB vs AAA vs $\mathrm{PBC}=4048 \mathrm{cGy}$ vs $4365 \mathrm{~Gy}$ vs 4873Gy, $P<0.05)$. PBC induced the enhanced trend of $\mathrm{D}_{\max }$ of PTV compared with other two algorithms (For $\mathrm{D}_{\max }$ of PTV, AXB vs AAA vs $\mathrm{PBC}=5897 \mathrm{cGy}$ vs $5904 \mathrm{~Gy}$ vs $6122 \mathrm{~Gy}$; $\mathrm{AXB}$ vs AAA $P=0.41$; $\mathrm{AXB}$ vs $\mathrm{PBC} P=0.06$; AAA vs PBC: $P=0.01$ ), and increased the $\mathrm{D}_{\text {mean }}$ of PTV in three algorithms with significant difference $\left(\right.$ For $\mathrm{D}_{\text {mean }}$ of PTV, AXB vs AAA vs $\mathrm{PBC}=5332 \mathrm{cGy}$ vs $5330 \mathrm{~Gy}$ vs 5785Gy, $P<0.05)$.

\section{$\mathrm{Cl}$ and $\mathrm{HI}$ of the PTV}

CI and HI of the PTV in 38 patients are shown in Table 4 and Figure 3. AXB algorithm achieved a similar plan conformity with other two algorithms (For CI, AXB vs AAA vs $\mathrm{PBC}=0.80$ vs 0.85 vs $0.71, P>0.05$ ) but slightly higher tend of target dose heterogeneity (For HI, AXB vs AAA vs $\mathrm{PBC}=0.20$ vs 0.14 vs $0.31, P>0.05$ ).

\section{Dosimetric distribution for the plan target volume}

The isodose distributions in the axial view at the isocenter and dose volume histogram for the same patient calculated from the three algorithms are presented in Figure 4. The PTV coverage of AXB plan was lower than that of the other two plans. It can be seen from Figure 4 that the $100 \%$ prescription dose line of the PBC algorithm includes the largest target area.

\section{Discussion}

The application of different algorithms in the radiotherapy physics plan has always been the research direction of scholars. $^{3,4,7-13}$ The AXB algorithm is similar to the Monte Carlo algorithm, the physical action of the ray in the medium was clearly simulated, and the linear Boltzmann linear transmission problem was solved in the discrete and multidimensional space. ${ }^{6}$ Many scholars have confirmed that the $\mathrm{AXB}$ algorithm was the closest to the Monte Carlo algorithm, ${ }^{10,14}$ the accuracy was higher than the AAA and PBC algorithms. ${ }^{15-17}$ The AAA algorithm is based on the superposition convolution technique, and the uniformity correction is considered in the calculation. ${ }^{9}$ The 
Table 2 The V100\% and V90\% of PTV of the three algorithms

\begin{tabular}{|c|c|c|c|c|c|c|}
\hline \multirow[t]{2}{*}{ Parameter } & \multicolumn{3}{|c|}{ Mean \pm SD (\%) } & \multicolumn{3}{|l|}{$P$-value } \\
\hline & AXB & AAA & PBC & AXB vs AAA & $A X B$ vs $\mathrm{PBC}$ & AAAvs PBC \\
\hline V $100 \%$ & $93.44 \pm 2.03$ & $95.54 \pm 0.47$ & $99.58 \pm 1.01$ & 0.21 & 0.00 & 0.02 \\
\hline V90\% & $99.66 \pm 0.73$ & $99.75 \pm 0.63$ & $99.83 \pm 0.49$ & 0.32 & 0.26 & 0.99 \\
\hline
\end{tabular}

Note: $P<0.05$ considered statistically significant.

Abbreviation: SD, standard deviation.

A

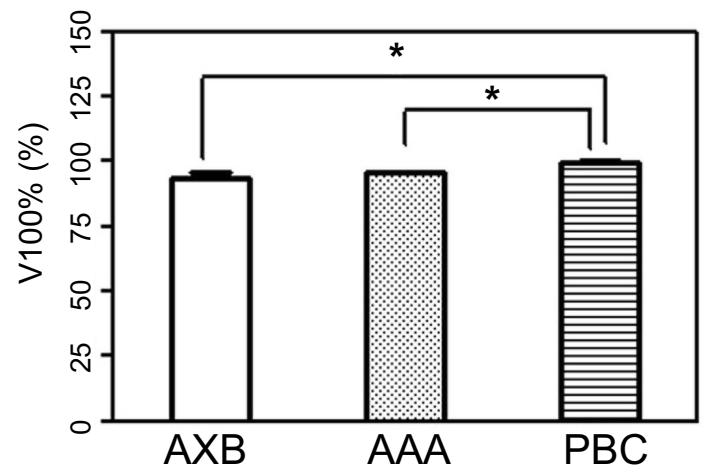

B

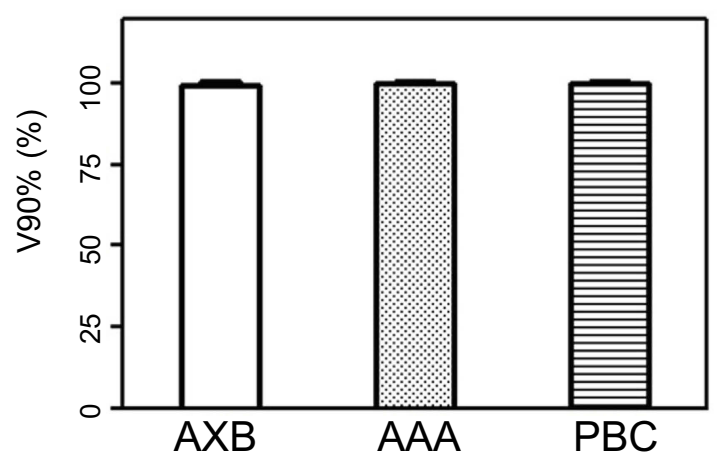

Figure I The comparison of the V100\% (A) and V90\% (B) of the PTV in the three algorithms. *means $P<0.05$.

Notes: V100\% was defined as the volume of the prescription dose line for $100 \%$ includes the volume of PTV. V $90 \%$ was defined as the volume of PTV contained in the prescription dose line for $90 \%$.

Abbreviation: SD, standard deviation.

Table $3 D_{\min }, D_{\max }$, and $D_{\text {mean }}$ of PTV

\begin{tabular}{|c|c|c|c|c|c|c|}
\hline \multirow[t]{2}{*}{ Parameter } & \multicolumn{3}{|c|}{ Mean \pm SD (cGy) } & \multicolumn{3}{|l|}{$P$-value } \\
\hline & $\mathbf{A X B}$ & AAA & PBC & AXB vs AAA & $A X B$ vs $P B C$ & AAA vs PBC \\
\hline$D_{\min }$ & $4048 \pm 327$ & $4365 \pm 231$ & $4873 \pm 306$ & 0.04 & 0.00 & 0.00 \\
\hline$D_{\max }$ & $5897 \pm 245$ & $5804 \pm 240$ & $6122 \pm 175$ & 0.41 & 0.06 & 0.01 \\
\hline$D_{\text {mean }}$ & $5332 \pm 116$ & $5330 \pm 132$ & $5785 \pm 166$ & 0.97 & 0.00 & 0.00 \\
\hline
\end{tabular}

Note: $P<0.05$ considered statistically significant.

Abbreviation: SD, standard deviation.

PBC algorithm is a pencil beam algorithm, which calculates dose distribution through a very narrow beam, each pencil takes into account the attenuation factor of different beams, even the method of equivalent tissue air ratio is used for non-uniformity correction. But the lateral nonuniformity correction is not considered in the $\mathrm{PBC}$ algorithm. $^{7}$

There is a strong correlation between tumor control probability and radiation dose in SBRT radiotherapy, accurate radiation dose to the target volume is very important. ${ }^{8}$ Therefore, it is necessary to adopt a more accurate dose calculation algorithm in radiotherapy physics plan. The present research showed that $\mathrm{V} 100 \%$ in group of the $\mathrm{PBC}$ algorithm was more than that in the group of the $\mathrm{AXB}$ and AAA algorithms, which indicated that the PBC algorithm significantly overestimates the dose of PTV in dose calculation. Meanwhile, the V100\% in the group of AAA algorithm was $3 \%$ larger than that in the group of AXB without statistical significance $(P=0.21)$. The results here were consistent with the previous results of Rana. ${ }^{13}$ Rana and his colleagues found that the $\mathrm{V} 100 \%$ in the group of AAA is $8.2 \%$ higher than that in the group of AXB algorithm in 16 cases of non-small cell lung cancer. It showed that the AAA algorithm would have an effect on target dose. For V90\%, the difference between AAA and PBC algorithm was not statistically significant compared with AXB algorithm. The value of $\mathrm{V} 90 \%$ in three algorithms was more than $98 \%$, which showed that $90 \%$ of the prescription dose lines obtained by the three algorithms can effectively encircle the target area. 
A

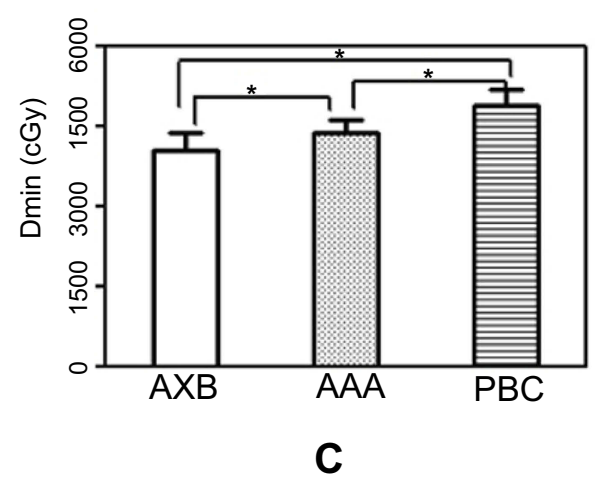

B

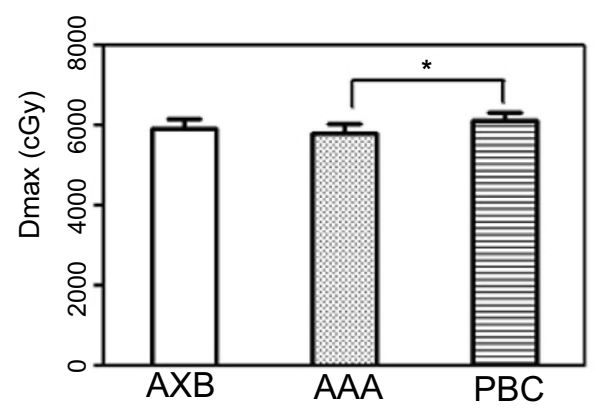

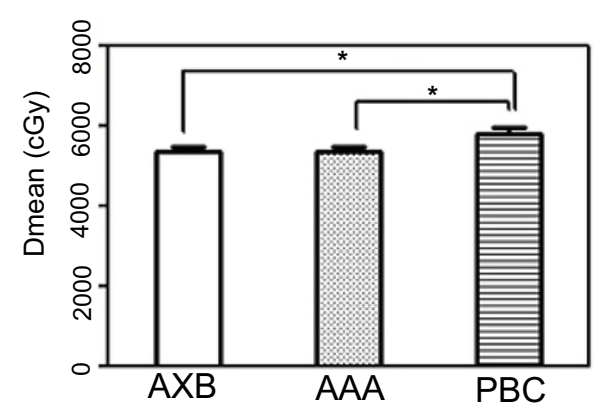

Figure 2 The comparison of $D_{\min }(\mathbf{A}), D_{\max }(\mathbf{B})$, and $D_{\text {mean }}(\mathbf{C})$ of PTV in the three algorithms. *means $P<0.05$.

Table $4 \mathrm{Cl}$ and $\mathrm{HI}$ of PTV

\begin{tabular}{|c|c|c|c|c|c|c|}
\hline \multirow[t]{2}{*}{ Parameter } & \multicolumn{3}{|l|}{ Mean士SD } & \multicolumn{3}{|l|}{$P$-value } \\
\hline & $\mathbf{A X B}$ & AAA & PBC & $A X B$ vs $A A A$ & $A X B$ vs $P B C$ & AAA vs PBC \\
\hline $\mathrm{Cl}$ & $0.80 \pm 0.08$ & $0.85 \pm 0.06$ & $0.7 I \pm 0.05$ & 0.14 & 0.01 & 0.00 \\
\hline $\mathrm{HI}$ & $0.20 \pm 0.07$ & $0.14 \pm 0.08$ & $0.3 I \pm 0.07$ & 0.13 & 0.08 & 0.02 \\
\hline
\end{tabular}

Note: $P<0.05$ considered statistically significant. Abbreviation: SD, standard deviation.

A

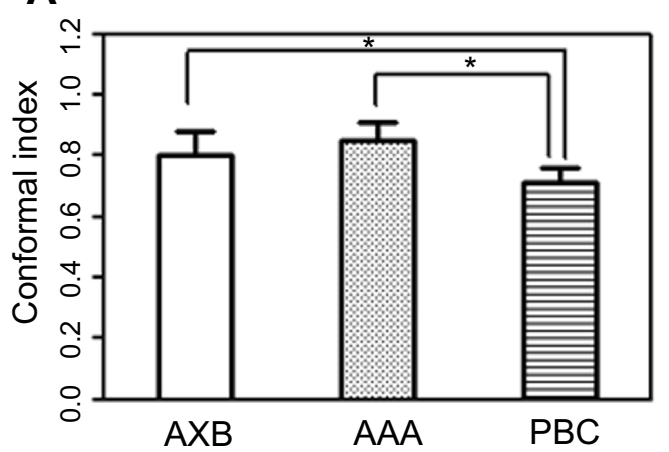

B

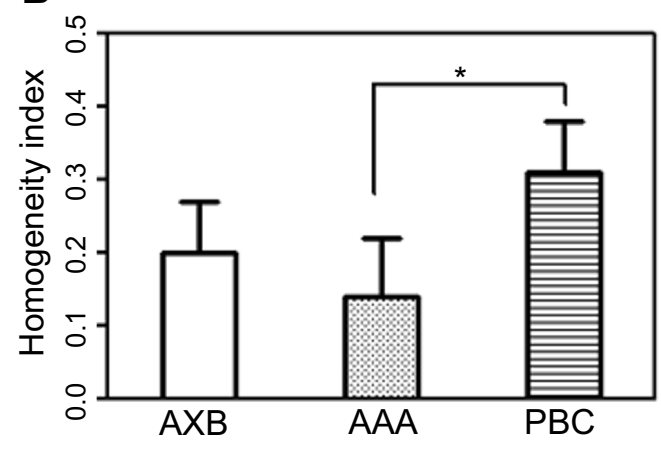

Figure 3 The comparison of $\mathrm{Cl}(\mathbf{A})$ and $\mathrm{HI}(\mathbf{B})$ of the PTV in the three algorithms. *means $P<0.05$. Abbreviations: SD, standard deviation; $\mathrm{Cl}$, conformal index; HI, Homogeneity index.

The present study also found that the trend of the $\mathrm{D}_{\text {min }}$, the $\mathrm{D}_{\max }$ and the $\mathrm{D}_{\text {mean }}$ for PTV was the same as that of V100\%. Compared with AXB, 20\% increase of $\mathrm{D}_{\min }$ in
PBC algorithm $(P=0.00)$ was found, $4 \%$ increase for $\mathrm{D}_{\max }$ $(P=0.06)$, about $8 \%$ increase for $\mathrm{D}_{\text {mean }}(P=0.00)$. All of these data also indicated that the PBC algorithm 

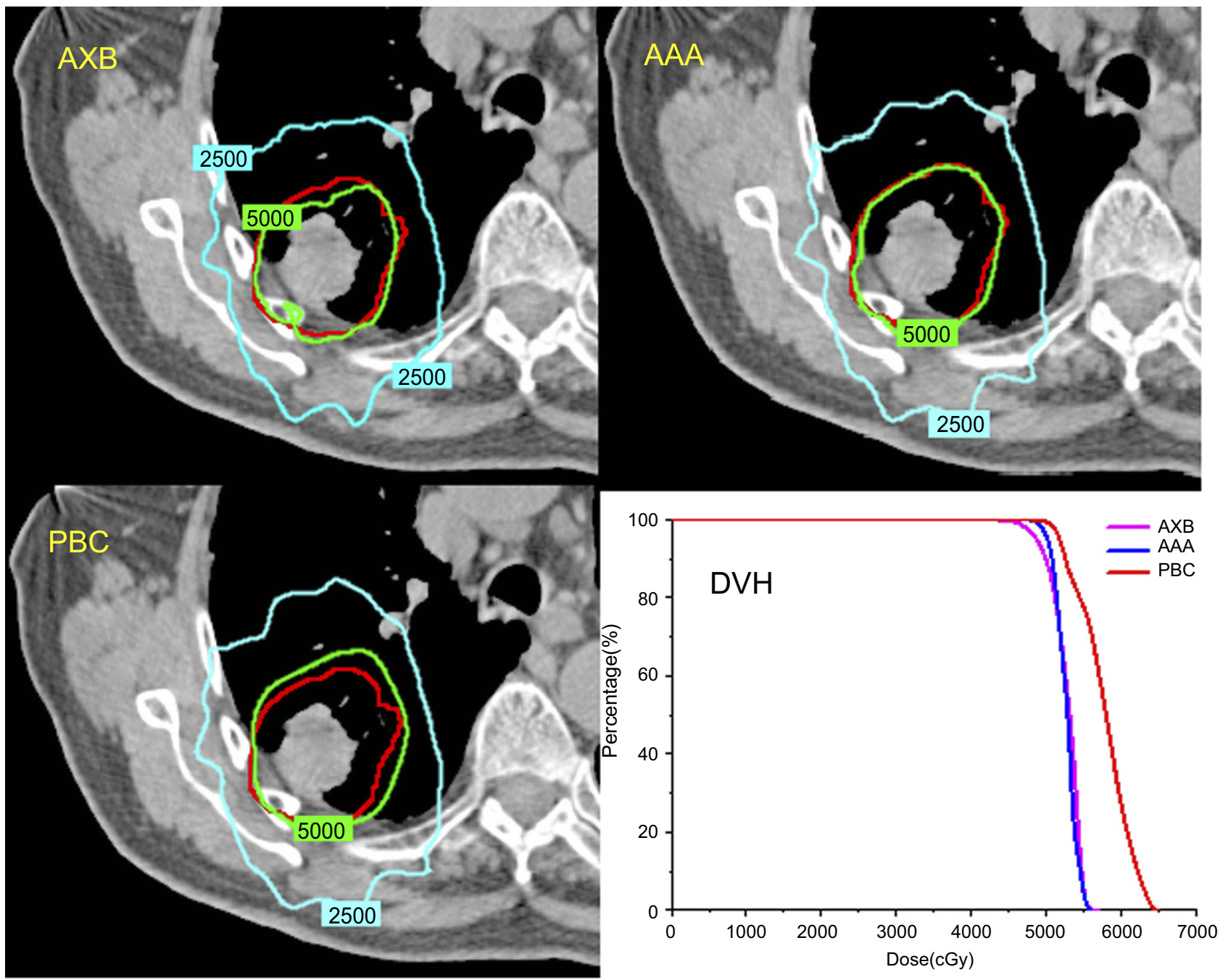

Figure 4 The isodose distributions in the axial view at the isocenter and dose volume histogram (DVH) of a patient. The red line was PTV, the green line is the isodose line of prescription dose $5000 \mathrm{cGy}(100 \%)$, and the light blue line is an isodose line of $2500 \mathrm{cGy}(50 \%)$.

significantly could overestimate the target dose. It was also found that the $\mathrm{D}_{\min }$ calculated by the AAA algorithm was about $8 \%(P=0.04)$ more that gained by the AXB algorithm, but there was no significant difference between the $D_{\text {max }}$ and the $D_{\text {mean }}$ between the two algorithms. These implied that the AAA algorithm does not significantly overestimate the target dose.

The CI for PTV in the group of the AAA was about $6 \%$ more than that in the AXB algorithm, and in the PBC algorithm was the smallest. The probable reason was overestimating the dose of the target area by the PBC algorithm which results in a larger volume size of the prescription dose. HI of PTV gained from PBC algorithm was maximum, which showed that the dose of the PTV of the PBC algorithm was the most heterogeneous. The foregoing results indicated that the PBC algorithm can significantly overestimate the target dose because the lung is considered as a homogeneous tissue, the AAA algorithm would also overestimate the dose of PTV. AXB can more authentically simulate the physical process of radiation in heterogeneous tissue.

\section{Conclusion}

The present study demonstrated that the $\mathrm{PBC}$ and the AAA algorithm could overestimate the dose of PTV for SBRT physics plan for non-small cell lung cancer compared to the AXB algorithm. Therefore, the AAA and PBC algorithms were inaccurate for the PTV calculation of the planned target area. In the clinical plan of SBRT for nonsmall cell lung cancer, the AXB algorithm should be suggested to improve the accuracy of the dose plan.

\section{Ethic statement}

This study has been approved by the Ethics Committee of Zhongnan Hospital of Wuhan University. 


\section{Acknowledgments}

This research was partially supported by Zhongnan Hospital of Wuhan University Science, Technology and Innovation Seed Fund (Grant No. ZNPY2016043), Natural Science Foundation of Hubei Province (Grant No. 2018CFB561) and Scientific Fund of Health Commission of Hubei Province (Grant No. WJ2019M264).

\section{Disclosure}

The authors declare no conflicts of interest in this work.

\section{References}

1. Chang JY, Senan S, Paul MA, et al. Stereotactic ablative radiotherapy versus lobectomy for operable stage I non-small-cell lung cancer: a pooled analysis of two randomised trials. Lancet Oncol. 2015;16 (6):630-637. doi:10.1016/S1470-2045(15)70168-3

2. Baumann P, Nyman J, Hoyer M, et al. Outcome in a prospective phase II trial of medically inoperable stage I non-small-cell lung cancer patients treated with stereotactic body radiotherapy. J Clin Oncol. 2009;27(20):3290-3296. doi:10.1200/JCO.2008.21.5681

3. Rana S, Rogers K, Pokharel S, Cheng C. Evaluation of acuros XB algorithm based on RTOG 0813 dosimetric criteria for SBRT lung treatment with RapidArc. J Appl Clin Med Phys. 2014;15(1):4474. doi:10.1120/jacmp.v15i1.4474

4. Zhou C, Bennion N, Ma R, et al. A comprehensive dosimetric study on switching from a Type-B to a Type-C dose algorithm for modern lung SBRT. Radiat Oncol. 2017;12(1):80. doi:10.1186/s13014-017-0816-x

5. Bezjak A, Bradley J, Gaspar L, Robert D, Papiez L, Gore E. Seamless phase I/II study of stereotactic lung radiotherapy (SBRT) for early stage, centrally located, non-small cell lung cancer (NSCLC) in medically inoperable patients. RTOG. 2012;813:1-75.

6. Liang X, Penagaricano J, Zheng D, et al. Radiobiological impact of dose calculation algorithms on biologically optimized IMRT lung stereotactic body radiation therapy plans. Radiat Oncol. 2016;11 (1):10. doi:10.1186/s13014-015-0578-2

7. Dorje T. Limitation of pencil beam convolution (PBC) algorithm for photon dose calculation in inhomogeneous medium. $J$ Cancer Treat Res. 2014;2(1):1-4. doi:10.11648/j.jctr.20140201.11
8. Liu H-W, Nugent Z, Clayton R, Dunscombe P, Lau H, Khan R. Clinical impact of using the deterministic patient dose calculation algorithm Acuros XB for lung stereotactic body radiation therapy. Acta Oncol (Madr). 2014;53(3):324-329. doi:10.3109/ 0284186X.2013.822552

9. Zhen H, Hrycushko B, Lee $\mathrm{H}$, et al. Dosimetric comparison of Acuros XB with collapsed cone convolution/superposition and anisotropic analytic algorithm for stereotactic ablative radiotherapy of thoracic spinal metastases. J Appl Clin Med Phys. 2015;16(4):181192. doi:10.1120/jacmp.v16i4.5493

10. Ping LX, Bao ZY, Hao WU, Zhen YH. Accuracy comparison of Acuros $\mathrm{XB}$ algorithm, anisotropic analytical algorithm and Monte Carlo algorithm in calculating doses of inhomogeneous tissues. Chin $\mathrm{J} \mathrm{Med}$ Phys. 2016;33(4):348-352. doi:10.3969/j.issn.1005-202X.2016.04.005

11. Zheng D, Zhang Q, Liang X, et al. Effect of the normalized prescription isodose line on the magnitude of Monte Carlo vs. pencil beam target dose differences for lung stereotactic body radiotherapy. J Appl Clin Med Phys. 2016;17(4):48-58. doi:10.1120/jacmp.v17i4.5965

12. Zheng D, Zhu X, Zhang Q, et al. Target dose conversion modeling from pencil beam (PB) to Monte Carlo (MC) for lung SBRT. Radiat Oncol. 2016;11(1):83. doi:10.1186/s13014-016-0661-3

13. Rana S, Rogers K, Lee T, Reed D, Biggs C. Verification and dosimetric impact of Acuros XB algorithm for stereotactic body radiation therapy (SBRT) and RapidArc planning for non-small-cell lung cancer (NSCLC) patients. Int J Med Phys Clin Eng Radiat Oncol. 2013;2 (01):6. doi:10.4236/ijmpcero.2013.21002

14. Han T, Mikell JK, Salehpour M, Mourtada F. Dosimetric comparison of Acuros $\mathrm{XB}$ deterministic radiation transport method with Monte Carlo and model-based convolution methods in heterogeneous media. Med Phys. 2011;38(5):2651-2664. doi:10.1118/ 1.3582690

15. Gagné IM, Zavgorodni S. Evaluation of the analytical anisotropic algorithm in an extreme water-lung interface phantom using Monte Carlo dose calculations. J Appl Clin Med Phys. 2007;8(1):33-46. doi:10.1120/jacmp.v8i1.2324

16. Han T, Followill D, Mikell J, et al. Dosimetric impact of Acuros XB deterministic radiation transport algorithm for heterogeneous dose calculation in lung cancer. Med Phys. 2013;40(5):051710. doi:10.1118/1.4802216

17. Fogliata A, Lobefalo F, Reggiori G, et al. Evaluation of the dose calculation accuracy for small fields defined by jaw or MLC for AAA and Acuros XB algorithms. Med Phys. 2016;43(10):5685. doi: $10.1118 / 1.4963219$
OncoTargets and Therapy

\section{Publish your work in this journal}

OncoTargets and Therapy is an international, peer-reviewed, open access journal focusing on the pathological basis of all cancers, potential targets for therapy and treatment protocols employed to improve the management of cancer patients. The journal also focuses on the impact of management programs and new therapeutic agents and protocols on patient perspectives such as quality of life, adherence and satisfaction. The manuscript management system is completely online and includes a very quick and fair peer-review system, which is all easy to use. Visit http://www.dovepress.com/ testimonials.php to read real quotes from published authors. 